\title{
Reply to GS Wand and L Oswald \\ Reply: Dissociable Hormonal, Cognitive, and Mood Responses to Neuroendocrine Challenge: Evidence for Receptor-Specific Serotonergic Dysregulation in Depressed Mood
}

\author{
Wim J Riedel*,', Tineke Klaassen', Eric Griez', Adriaan Honig', Paul Menheere' and Herman M Van Praag' \\ 'Brain \& Behaviour Institute, Maastricht University, Maastricht, The Netherlands
}

Neuropsychopharmacology (2003) 28, I0 I2-10 I3, advance online publication, 19 March 2003; doi: I 0. I 038/sj.npp. I 300076

Sir

We have read the letter by Wand and Oswald (2003) with great interest, but respectfully disagree with their position (1) that a difference in acth/cortisol ratio explains our observation (Riedel et al, 2002) of a blunted cortisol response to 5-HT1A challenge in depressed patients, rather than a central downregulation of 5-HT1A receptors, and (2) that the differential effect of $m$-cpp on cortisol levels reflects different expressions of 5-HT modulation on hypothalamic (hormonal) and amygdalo-frontal (mood) pathways. However, we do take their last point very seriously that (3) future studies should take into account peripheral as well as central effects of serotonergic agents on the HPA axis.

(1) Wand and Oswald state that our data show that m-cpp and ipsapirone have different effects on ACTH, and consequently they infer that there must be a different (peripheral) pathway through which $\mathrm{m}$-cpp alters cortisol secretion.

Briefly, we think Wand and Oswald have misread the graphs by ignoring the information contained in the standard errors. There is in fact no overall difference between the level of acth responses to m-cpp and ipsapirone when the distribution of the responses is taken into account. Needless to say, this is what is always incorporated in statistical testing. It is true, as in all human hormonal challenge experiments, that the distributions of responses are skewed. In the article, this is acknowledged in the statistics section by explicitly stating that for statistical testing the hormonal values were subjected to logarithmic transformation. Rather than show plots of logarithmic hormonal values however, we chose to show plots of the mean+standard errors of the raw (difference) scores to provide the reader with as much information as possible.

(2) Furthermore, Wand and Oswald seriously misinterpret or misquote us when they write: 'The authors suggest that the differential effect of m-cpp on cortisol levels reflects different expressions of 5-HT modulation on hypothalamic

*Correspondence: Dr WJ Riedel, E-mail: willem.j.riedel@gsk.com (hormonal) and amygdalo-frontal (mood) pathways (Stahl, 1998).'

In fact, this is not what we wrote, neither is it what we wanted to suggest on p. 366:

'The effects of $\mathrm{m}$-CPP and ipsapirone most probably reflect different expressions of serotonergic modulation in hypothalamic (hormonal), amygdalo-frontal (mood) and hippocampal (memory) pathways, although it must be noted here that these associations are only well established in the case of endocrine effects mediated via serotonergic modulation of the hypothalamus.' (Riedel et al, 2002)

So, this statement concerned the explanation of the pattern of all effects of the two substances, not just on cortisol! Therefore, their criticism misses the essence of the article, which never intended to state that all other effects than those (hypothalamically mediated) are dependent on cortisol. In fact, the title of the article suggests that the effects of $\mathrm{m}$-cpp and ipsapirone on mood and cognition are dissociable (NOT mediated through the hypothalamic pathway!!) from those on cortisol. Any putative alternative mechanism to explain cortisol effects would leave those observations primarily intact.

(3) The point to take peripheral as well as central effects of serotonergic agents on the HPA axis into account in future studies is well taken. However, at present it is unclear as to how this should be dealt with in a more direct manner than using the calculation of the acth/cortisol ratio as a rather indirect inference of peripheral $v s$ central influences of drugs on cortisol. Wand and Oswald implicitly suggest that the possible peripheral effects of $\mathrm{m}$-cpp could be because of peripheral 5-HT4 action directly on the adrenal gland and consequently cortisol release (Delarue et al, 1998; Contesse et al, 2000). Although it is doubtful that m-cpp has significant 5-HT4 affinity, there are no in vivo human experimental data to substantiate this. An m-cpp challenge in conjunction with a 5-HT4 antagonist to block putative peripheral influences of $\mathrm{m}$-cpp directly on the adrenal gland could make up a dedicated experiment to tackle this question. Central effects of 5-HT4 manipulations on cognitive and behavioural variables should also be mon- 
itored in such an experiment because of their possible memory-enhancing properties (Eglen et al, 1995; Hegde and Eglen, 1996).

We maintain the position that mechanistic conclusions can be drawn from challenge protocols, but agree that these are always open to refinement; in this case measures taken to control for direct peripheral influences on the hormonal outcome variables have the potential to improve these challenge protocols.

\section{REFERENCES}

Contesse V, Lefebvre H, Lenglet S, Kuhn JM, Delarue C, Vaudry H (2000). Role of 5-HT in the regulation of the brain-pituitaryadrenal axis: effects of 5-HT on adrenocortical cells. Can J Physiol Pharmacol 78: 967-983.
Delarue C, Contesse V, Lefebvre H, Lenglet S, Grumolato L, Kuhn JM et al (1998). Pharmacological profile of serotonergic receptors in the adrenal gland. Endocr Res 24: 687-694.

Eglen RM, Wong EH, Dumuis A, Bockaert J (1995). Central 5-HT4 receptors. Trends Pharmacol Sci 16: 391-398.

Hegde SS, Eglen RM (1996). Peripheral 5-HT4 receptors. FASEB J 10: 1398-1407.

Riedel WJ, Klaassen T, Griez E, Honig A, Menheere PPCA, Van Praag HM (2002). Dissociable hormonal, cognitive and mood responses to neuroendocrine challenge: evidence for receptorspecific serotonergic dysregulation in depressed mood. Neuropsychopharmacology 26: 358-367.

Wand GS, Oswald L (2003). Re: dissociable hormonal, cognitive and mood responses to neuroendocrine challenge: evidence for receptor-specific serotonergic dysregulation in depressed mood. Neuropsychopharmacology 28: 1006. 